\title{
Proactive Guidance for Accurate UAV Landing on a Dynamic Platform: A Visual-inertial Approach
}

\author{
Ching-Wei Chang ${ }^{1}$, Li-Yu Lo ${ }^{2}$, Hiu Ching Cheung ${ }^{1}$, Yurong Feng ${ }^{2}$, An-Shik Yang ${ }^{3}$, Chih-Yung WEN ${ }^{2}$, and \\ Weifeng ZHOU 4,*
}

1 Department of Mechanical Engineering, The Hong Kong Polytechnic University, Kowloon, Hong Kong; chingwei.chang@connect.polyu.hk (C.-W.C.); hiu-ching-athena.cheung@connect.polyu.hk (H.H.C.)

2 Department of Aeronautical and Aviation Engineering, The Hong Kong Polytechnic University, Kowloon, Hong Kong; liyu.lo@connect.polyu.hk (L.-Y.L.); yurong.feng@connect.polyu.hk (Y.F.); chihyung.wen@polyu.edu.hk (C.-Y.W.)

3 Department of Energy and Refrigerating Air-Conditioning Engineering, National Taipei University of Technology, Taipei, 10608, Taiwan; asyang@ntut.edu.tw (A.-S.Y.)

4 School of Professional Education and Executive Development, The Hong Kong Polytechnic University, Kowloon, Hong Kong; chandler.zhou@cpce-polyu.hk (W.Z.)

* Correspondence: chandler.zhou@cpce-polyu.edu.hk; Tel.: +852 37460127

\begin{abstract}
This work aims to develop an autonomous system for the unmanned aerial vehicle (UAV) to land on a moving platform such as the automobile or marine vessels, providing a promising solution for a long-endurance flight operation, a large mission coverage range, and a convenient recharging ground station. Different from most state-of-the-art UAV landing frameworks which rely on UAV's onboard computers and sensors, the proposed system fully depends on the computation unit situated on the ground vehicle/marine vessel to serve as a landing guidance system. Such novel configuration can therefore lighten the burden of the UAV and computation power on the ground vehicle/marine vessel could be enhanced. In particular, we exploit a sensor fusion-based algorithm for the guidance system to perform UAV localization, whilst a control method based upon trajectory optimization is integrated. Indoor and outdoor experiments are conducted and the result shows that a precise autonomous landing on a $43 \times 43 \mathrm{~cm}$ platform could be performed.
\end{abstract}

Keywords: UAV; VTOL; Object Tracking; Deep Learning; Sensor fusion; Kalman Filter; Autonomous Landing; Optimal Trajectory.

\section{Introduction}

In recent years, the development and application of unmanned aerial vehicles (UAV) have been rapid. For instance, agriculture industries, construction sectors, commercial delivery corporates, and many others are now eager to adopt aerial robots to boost their working efficiency [1-3]. Nevertheless, the limited payload of a UAV determines a fixed battery capacity, which requires frequent landing operations for battery replacement or recharging. The need for an autonomous landing system of UAVs on moving platforms is hence enormous, where the operation efficiency would be further increased.

In order to address such an issue, this work has set out to design a system framework for a quadrotor UAV to perform landing on unmanned ground vehicles (UGV), where several pioneer works could be seen $[4,5]$. For instance, DHL's AMP Electric Vehicle has been testing the applicability of UAVs pairing with delivery trucks, where the aerial robots deliver parcels that are outside of the main delivery route of the truck. During the operation, the aerial vehicles are positioned on top of the delivery truck. After loaded, the UAV will schedule the route to the delivery point via GPS and take off from the moving track. In the meantime, the truck will continue its rounds. After a successful delivery, the UAV will fly back to the truck for its next delivery run, where it can also recharge its battery wirelessly [6]. Obviously, with such an autonomous landing system, the delivery 
efficiency of a single UAV can be increased. Similar technology is also under preliminary research in Amazon Prime Air [7].

The system can be further applied to marine purposes. With a robust and reliable autonomous landing system, UAVs can conduct missions on a moving ship, such as auto marine condition surveying, including the detection of detailed air quality or weather conditions above the sea $[8,9]$. With an automatic UAV system equipped on ships, the offshore marine devices can be deployed in a much faster manner [10, 11]. Meanwhile, the UAV system can help the maritime safety department to monitor any unidentified boat or ship in the open sea in a much faster and safer manner [12,13]. Additionally, it can also be of great assistance in maritime search and rescue (SAR) mission to assist any boat or ship under threat [14-17]. The landing system can also be seen in aerial operations. The test in [18], for example, UAVs carried by a bigger flying platform can greatly reduce the time to reach the target and dramatically increase the operation range of UAVs.

In terms of the utilized sensors in this research scope, several variations can be observed. The study in [19] utilized a DJI F450 drone equipped with an infrared (IR) camera and a laser ranging sensor, in which IR beacons are installed at a heaving platform. This method has the advantage of enabling the landing during the nighttime or low light environment. However, the low resolution of small size onboard IR camera has strictly limited the sensing distance, making the detection result unreliable in middle and long ranges. Meanwhile, the small angle of view of the onboard IR camera imposes a tight restriction on the heading control of the UAV. Any disturbance that affects the heading of the UAV can cause the beacon out of view and lead to a termination of the landing process. Moreover, in [20], the authors tried to land a UAV on top of a moving ground vehicle with an AprilTag marker on the landing pad. Two cameras are installed on the UAV to detect the marker from far and near. Yet, carrying two cameras onboard a UAV will significantly reduce its payload and increase the power consumption. Additionally, the hardware platform in [21] utilized a DJI Matrice 100 quadrotor, which equipped a DJI Guidance System including the DJI Zenmuse X3 Gimbal and Camera system, the SF30 altimeter, and the DJI Manifold onboard computer. This research has demonstrated its feasibility after conducting a field test of UAV landing on a moving vehicle. Nonetheless, the approach is deemed to be nonapplicable in most scenarios, as it might overload most of the quadrotors with the onboard equipment. Similarly, other research proposed in [22, 23] also suffers from the overweighted payload onboard.

From above, many of the proposed works are based upon vision-based systems. However, such system might suffer from accuracy issues, as conventional computer vision techniques were applied in most systems. Therefore, utilizing object detection techniques to conduct position estimation is considered one great alternative to increase precision. The development of object detection has received numerous attentions for the last decades and the research outputs have been significant. Particularly, learning-based approaches are deemed to be the breakthrough in the field, such as work presented in [24] and [25]. As for vision-based robotics systems, several works integrating the foregoing topic could be seen. For instance, YOLOv4 was applied to develop an autonomous UAV system for E\&M devices in [26], whereas SVM for a quadrotor to track and follow a moving target was utilized in [27]. In addition to the above, the deep learning algorithm-based tracker was further adopted to conduct a surveillance mission on a UAV system [28]. Apart from that, severe motion blur could still be seen in most UAV landing work, and this was induced by the installation of cameras on UAV. Notably, the most proposed configuration in prior research works might also result in the limited field of view (FoV).

The control module of the landing process is also deemed one major module for a complete landing system. Particularly, the trajectory to descent to the landing pad is important, which should be varied according to the configuration of UAVs. In [29], two stages of landing were designed, 1 . approaching from long range and 2. landing after hovering above the landing platform. Meanwhile, a stage for the UAV to land after the approaching was specifically developed - yet there was no descending trajectory for the UAV to follow. Such a preliminary landing approach could then lead to unstable landing, 
as external factors such as wind disturbance could have a certain impact. To deal with such a problem, the study presented in [30] specifically focused on the controller development for wind disturbance rejection during the landing process. Yet, their proposed system was considered compromising in certain scenarios, as it purely relied on artificial pattern tags for the perception module. Therefore, in the current work, an optimized landing trajectory will be included and addressed so that the robustness of the system could be ensured to some extent.

In this study, inspired by the instrument landing system (ILS) in the real-world aviation industry, a UAV landing framework based upon a guidance system sitting on a UGV is proposed. In which, we have proposed a sensor-fusion and estimation method of multiple sources, including the ArUco marker, YOLO object detector, stereo depth information, and IMU information. A set of depth camera is mounted at the landing platform, as shown in Figure 1, and an ArUco tag is attached on a square pad at the front of the UAV. With this design, it is expected that the capacity of the computation unit can be extensively distinguished from those UAV configurations with a companion computer onboard. In the proposed system, a finite state machine is designed to track and control the landing process. This state machine includes four stages, which are 1. GPS following, 2. Vision position following, 3. Ground-effect free trajectory and 4. Shutting down. In particular, the state of ground-effect free trajectory signifies the generation of optimized trajectories for the UAV to track and follow. It is deemed that the elucidated framework could provide a faster, smoother, and safer landing than the previous works.

The innovation of this research includes 1. promoting a new installation method for the auxiliary equipment like the camera and the onboard computer on the moving platform such as USV/UGV instead of the UAV itself. This arrangement can minimize the demand of the UAV payload and simultaneously maximize the usage of the computational power, 2. proposing the application of ground-effect free trajectory into the auto landing system which shows a significant improvement from the ordinary vertical landing path of other researches, 3. firstly fusing the position estimation results from IMU, YOLO, and ArUco for automatic landing systems, in order to simultaneously achieve high estimation precision and high updating rate, and 4. specially designing a Finite State Machine of UAV to land on a moving platform for the consideration of the robustness and safety.

The remainder of this paper is structured as follows: Section 2 provides the detailed system architecture, including the vision-based position detection, the UAV position estimation method, the design of finite state machine, and the development of trajectory planner free from the ground effect; Section 3 describes the experimental platform and test environment; Section 4 then presents the experimental results and discussion; and Section 5 concludes this work. 


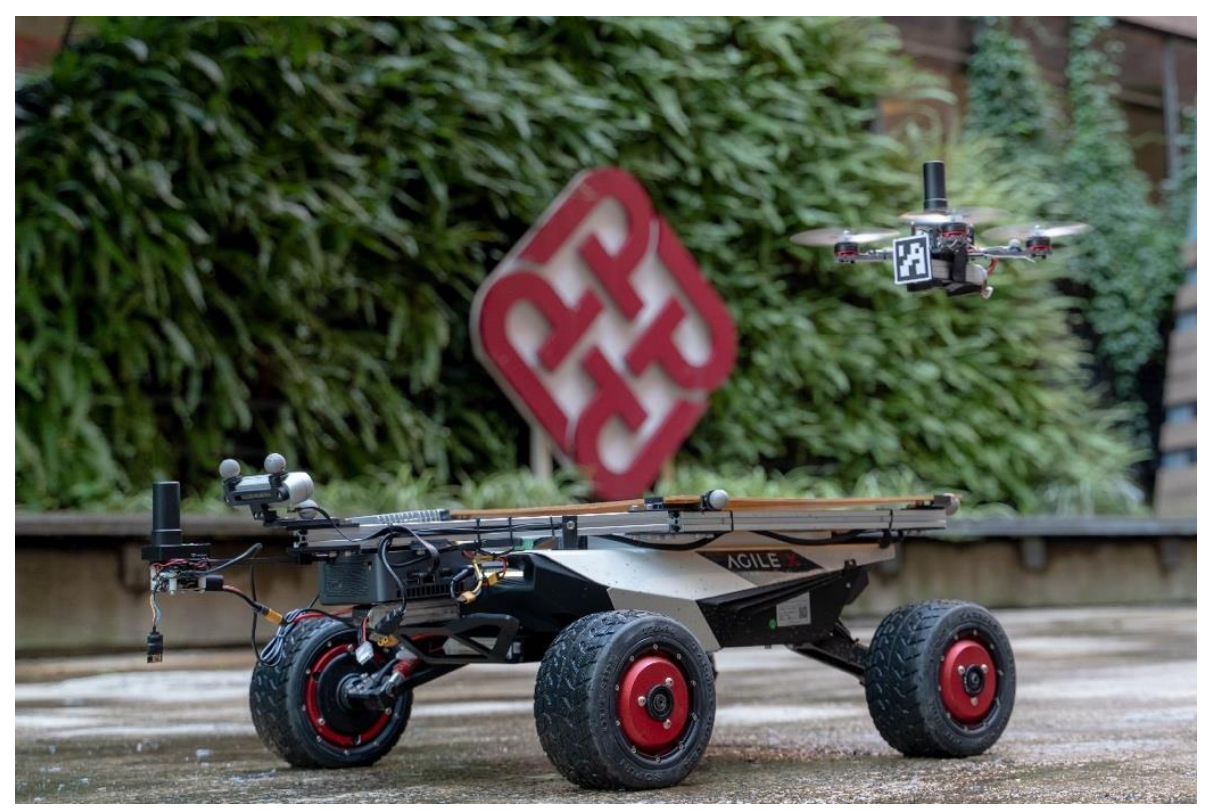

Figure 1. Prototype of the proactive guidance UGV and the UAV.

\section{System Architecture}

The landing System consists of several modular components, including:

1. Vision-based Position Detection, 2. UAV Position Estimation, 3. Finite State Machine, and 4. Ground-Effect Free Trajectory Planner. The relationship of each module is presented in Figure 2. This modular framework provides the availability of using different operating frequencies in independent modules and is easy to be modified or replaced with other algorithms.

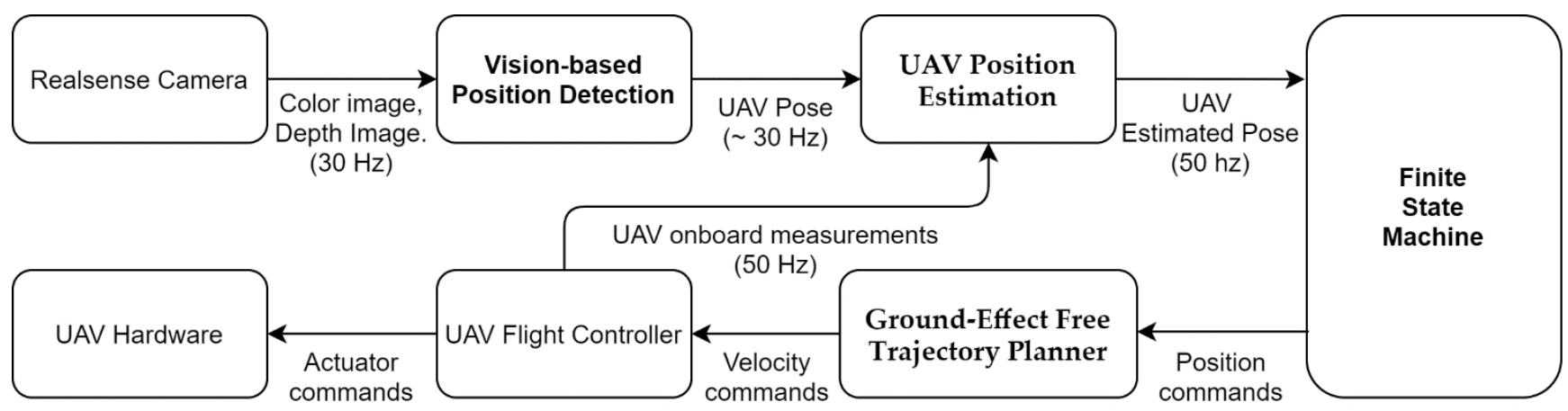

Figure 2. The system architecture of the autonomous landing system.

\subsection{Vision-based Position Detection}

In the proposed system, computer vision technologies are applied to perform visionbased landing guidance. To achieve this, the system must obtain the capabilities of perceiving an aerial vehicle in a 3D world, which can act as a feedback signal for the control system. Particularly, computer vision markers, as well as a learning-based detector, are adopted to conduct the task.

Specifically, ArUco markers are exploited and attached at the front of the quadrotor. With the high color contrast, the marker could be easily detected from the landing platform. By further solving the PnP problem (Perspective-n-Point), the relative position of the ArUco marker from the camera could be known. The inferred position information is then processed to acquire the position of the UAV. The major advantage of utilizing an ArUco marker is that it provides an extremely high update rate at a certain level of accuracy, yet the system suffers severely from frequent detection loss under occasional motion 
blur while conducting aggressive maneuver of the UAV. When the vehicle is relatively far, or has severe illumination problems due to unstable weather, the small marker size becomes the major limitation.

Therefore, to increase the robustness, apart from ArUco, the state-of-the-art YOLOv4 algorithm is further deployed, where the loss of detections could be considerably compensated. To apply YOLOv4, the foremost and most crucial work is to establish a meticulously labeled dataset and conduct model training to generate a custom model. Accordingly, the dataset should consist of diverse images of the UAV under different sizes, angles, resolutions, backgrounds, and illuminated conditions. Despite being relatively computationally demanding, it is deemed that the high accuracy and robustness detector can increase the stability of the proposed system. Furthermore, in real application scenarios, the moving platforms such as automobiles or marine vessels are capable of carrying computation facilities with powerful GPUs. It is deemed that YOLOv4 can perform UAV detection in real time, giving the system is with a sufficiently high update rate.

During a landing process, both ArUco and YOLO module first conduct predictions on the streaming frame. The ArUco detector will then generate a 3D position information of the observed ArUco marker, while the YOLO model will produce a Region of Interest (ROI) that possibly contains the aerial vehicle. For the retrieved result from the ArUco marker, the information is in the body frame, and thus, the 3D position of the ArUco in the world frame is then calculated by:

$$
\left[\begin{array}{c}
X_{i}^{W} \\
1
\end{array}\right]=T_{B}^{W}\left[\begin{array}{c}
X_{i}^{B} \\
1
\end{array}\right], \quad T_{B}^{W} \in S O(3)
$$

in which, where $X_{i}^{B}$ is the object position vector in body frame $(B)$, whereas $X_{i}^{W}$ is the 3D object state vector in the world frame $(W) . T_{B}^{W}$ is the transformation matrix between the body frame and the world frame.

As for the object detection model, the 2D bounding box is further integrated with the stereo reconstruction techniques. The depth of the pixels proximate to the center of the $\mathrm{ROI}$ is then obtained followed by the calculation of 3D position relative to the camera pose. To further acquire the 3D position information in the world frame, coordination transformation is then applied:

$$
\left[\begin{array}{c}
X_{i}^{W} \\
1
\end{array}\right]=T_{B}^{W} T_{C}^{B}\left[\begin{array}{c}
X_{i}^{C} \\
1
\end{array}\right], \quad T_{B}^{W}, T_{C}^{B} \in S O(3)
$$

where $X_{i}^{C}$ is the object position vector retrieved in the camera frame.

From the limited sensor available, we further filter the two different estimations in order to retrieve the final optimal estimation. The below section further discusses the detail of the filter-based estimation.

\subsection{UAV Position Estimation}

From the last section, the Vision-based Position Detection module detects and calculates the UAV's position in the world frame $(W)$. However, due to the limitation of camera frame rate (Max $30 \mathrm{~Hz}$, and the object is not always detectable), computational power, and sudden loss of visualization, the reliability of the position reference is not high enough for UAV's control in some circumstances. Furthermore, having a faster estimating rate of the position will also increase the response of the flight controller to provide better flying performance. To deal with these problems, a sensor fusion of multiple sources of position input (ArUco and Yolo Depth), and a position estimation approach is developed for the system.

To increase the updating rate and enhance the reliability of the position estimation, information from the onboard controller of the UAV will be collected and implemented into the position estimating system alongside the position information from ArUco and Yolo Depth. In this approach, a Kalman filter (KF) is designed for this position estimation task of a flying UAV. The UAV's state vector is written as, $s=\left[p_{x}, p_{y}, p_{z}, v_{x}, v_{y}, v_{z}\right]^{T}$, where $p_{x}, p_{y}, p_{z}$ are the position, and $v_{x}, v_{y}, v_{z}$ are the velocity according to the $x, y, z$ 
coordinate in the world frame $(W)$. The UAV's state in the system is predicted with the model:

$$
s_{k}=A_{k} s_{k-1}+w_{k}
$$

where $s_{k}, w_{\mathrm{k}}$, and $A_{k}$ are the state of the system, the processing noise (which is assumed to be a white Gaussian noise), and the state transition matrix of the flying UAV, respectively. The measurement model of the system is also considered with white Gaussian noise $v_{k}$, and is expressed as:

$$
z_{k}=H\left(s_{k}\right)+v_{k}
$$

In the proposed system, the measurement can be either from the vision system or the onboard sensors. However, due to the different updating rates of the ArUco module, YOLO module, and IMU in the flight controller, data is not synchronized. To overcome this problem, the first come first serve algorithm is adopted. At every update stage, the filter updates the velocity measurements from the flight controller (the highest updating rate), and then checks the state from the vision system. If there are new updated position data, the filter will also update the measured position result. While the system retrieves two measured positions at the same step, it will use the result from the vision system as measurement input.

According to the structure of the Kalman filter, the system contains two modules, which are the prediction module and the measurement-correction module. The two modules will alternate continuously. During the predicting stage, the state of the UAV will be first predicted by Equation (3), along with calculation of the uncertainty of the system, the covariance $C_{k}$, as:

$$
C_{k}=A_{k} C_{k-1} A_{k}^{T}+Q,
$$

where $Q$ is also a random Gaussian noise. Following with the measurement-correcting stage, the Kalman gain $K_{k}$ will be first calculated as follow:

$$
K_{k}=C_{k} H^{T}\left(H C_{k} H^{T}+Q\right)^{-1} .
$$

At last, the output state $s_{k}^{-}$and the covariance $C_{k}^{-}$are corrected with the Kalman gain in the following equations:

$$
\begin{gathered}
s_{k}^{-}=s_{k}+K_{k}\left(z_{k}-H s_{k}\right), \\
C_{k}^{-}=\left(I-K_{k} H\right) C_{k} .
\end{gathered}
$$

\subsection{Finite State Machine}

The finite state machine (FSM) module aims to help the recovering and landing stage of an entire flying mission. At the very beginning of the state machine, the state of the UAV is assumed to be under hovering mode waiting for landing. There is a total of four stages (states), including:

1. Following (with GPS),

2. Precise following (with the vision position estimation),

3. Ground-Effect Free Trajectory following,

4. Shutdown.

Figure 3 describes the logical connection of each state in the finite state machine and the criteria of state changing. The following subsections provides the detail. 


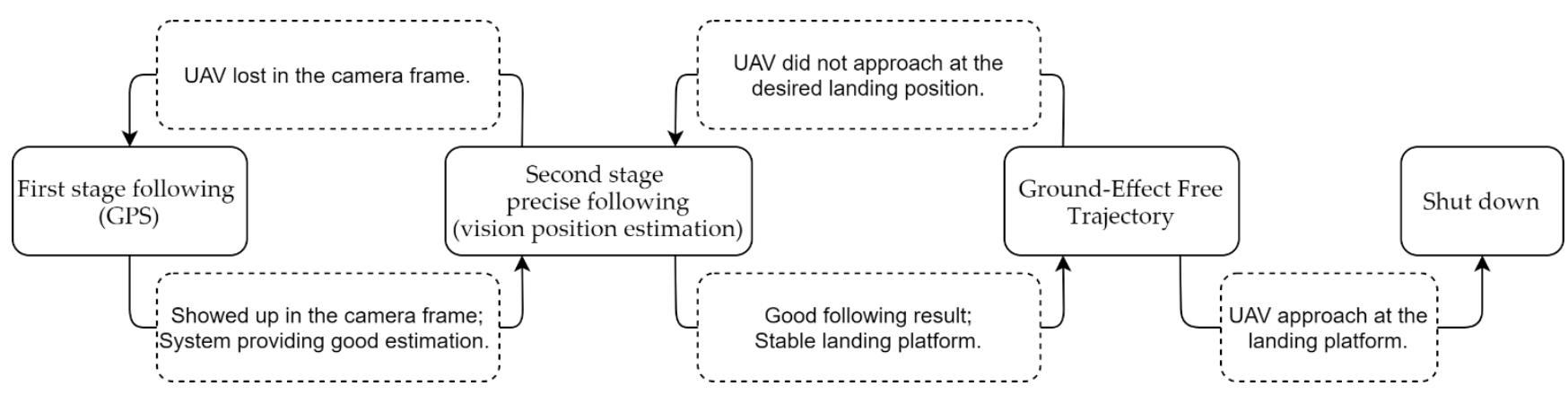

Figure 3. The framework of the finite state machine.

\subsubsection{First stage - following (GPS)}

In this first state, UAV is commanded to follow the landing platform with a preprogrammed distance and height difference. These parameters differ for different UAV models. The main purpose of this state is to use the GPS, which has limited positioning accuracy, to lead the UAV to enter the field of view $(\mathrm{FoV})$ of the autonomous landing system. Whenever the positioning estimation module starts to provide a reasonable (or convergence) reading of the position, it will trigger the state machine to switch to the next stage.

\subsubsection{Second stage - precise following (vision position estimation)}

After switching to this state, the FSM started passing velocity but not position commands toward the UAV's flight controller. The velocity setpoints are generated by the FSM's internal PID position (include $x, y, z$, and yaw) controller using the position reference from the position estimation module. The UAV will keep following at the same desired position as the first stage but controlled through the landing system without any GPS information. The goal of this stage is to ensure the stability of position (controlling and waiting for the landing platform to be ready for approaching. When the position difference between the landing platform and the UAV is in the desired domain, the stage will then proceed to the following motion. Not to mention, if the UAV's visibility is lost in the vision system and no position estimation result is provided, the system will rapidly move the stage backward to guide the UAV through GPS.

\subsubsection{Third stage - Ground-Effect Free Trajectory}

The purpose of this stage is to do the approaching maneuver in a smooth and safe trajectory, which generates less ground-effect compared to landing vertically. A detailed description of the method will be described in section 2.4. When the UAV meets the landing position, the state machine will then switch to the last stage. Several failsafe conditions are considered while the UAV is doing this approaching maneuver, which is: 1 . the divergence of the UAV's position with the desired trajectory, 2 . The failure of remaining altitude of the UAV to meet the final approach's requirement, and 3. the amount of overshoot of the UAV's position to the landing platform. If any of the criteria is out of bound, the state machine will immediately switch back to the previous stage and rapidly separate the UAV from the landing platform to keep a safe distance.

\subsubsection{Fourth stage - Shutdown}

The final stage is used to ensure the safety and stability of the UAV upon its contact with the landing platform. While the UAV entered the landing platform, ground-effect will start to affect the stability of the UAV. Whenever the UAV moves toward the landing platform within a reasonable altitude difference $(<5$ centimeters in this study) above the landing pad, the FSM will command the UAV to drastically reduce the throttles of the motors until all of them being shut down and the UAV lands solidly on the platform.

\subsection{Ground-Effect Free Trajectory Planner}

In this section, the landing trajectory planner will be introduced. There are two main purposes of this trajectory planner, which are: 1 . ensuring the smoothness and 
continuity of the approaching maneuver, 2. minimizing the instability caused by the ground effect.

Based on the work in [31], in which a super computational efficient optimal spatialtemporal trajectory planner has been proposed, we develop our method to guarantee the smoothness throughout the approaching and landing maneuver. This method can improve the computational efficiency, which enables this planner to generate a trajectory within a few microseconds. Besides, by setting up the parameters with the trajectory generator, physical actuation constraints of the UAV can be easily secured.

To reduce the significant ground effect and aerodynamic drag from the UAV, the desired trajectory is expected to reduce its' vertical only movements. In the proposed trajectory, the UAV is expected to rapidly reduce its altitude in open air before gliding onto the landing pad to prevent the possibility of causing the ground effect. Therefore, the Brachistochrone curve, which has the characteristic of using the shortest time to move an object with gravity from a higher point to the lower destination is introduced to this trajectory planner. The proposed trajectory will consider the initial state of the UAV and the landing pad's dynamic to generate a series of discrete waypoints according to the Brachistochrone curve.

\section{Experimental Architecture and Discussion}

To validate the proposed UAV system, both indoor and outdoor experiments are carried out. In this section, the detailed system architecture and the test environment are introduced

\subsection{Landing Platform Hardware Design}

In the experiments, a small Unmanned Ground Vehicle (UGV) Scout Mini (AgileX Robotics, China) is chosen to be the moving platform of the system. Figure 4 shows the setup of the UGV. On top of the vehicle an acrylic square pad $(43 \times 43 \mathrm{~cm})$ was mounted directly on the top, and a set of Intel RealSense D455 stereo camera (Intel Corporation, USA) was mounted in front of the landing plate with an upward-facing angle of $20^{\circ}$. The camera was also connected to an Intel NUC minicomputer (NUC8i7BEH, Intel Corporation, USA) for image collecting and processing.

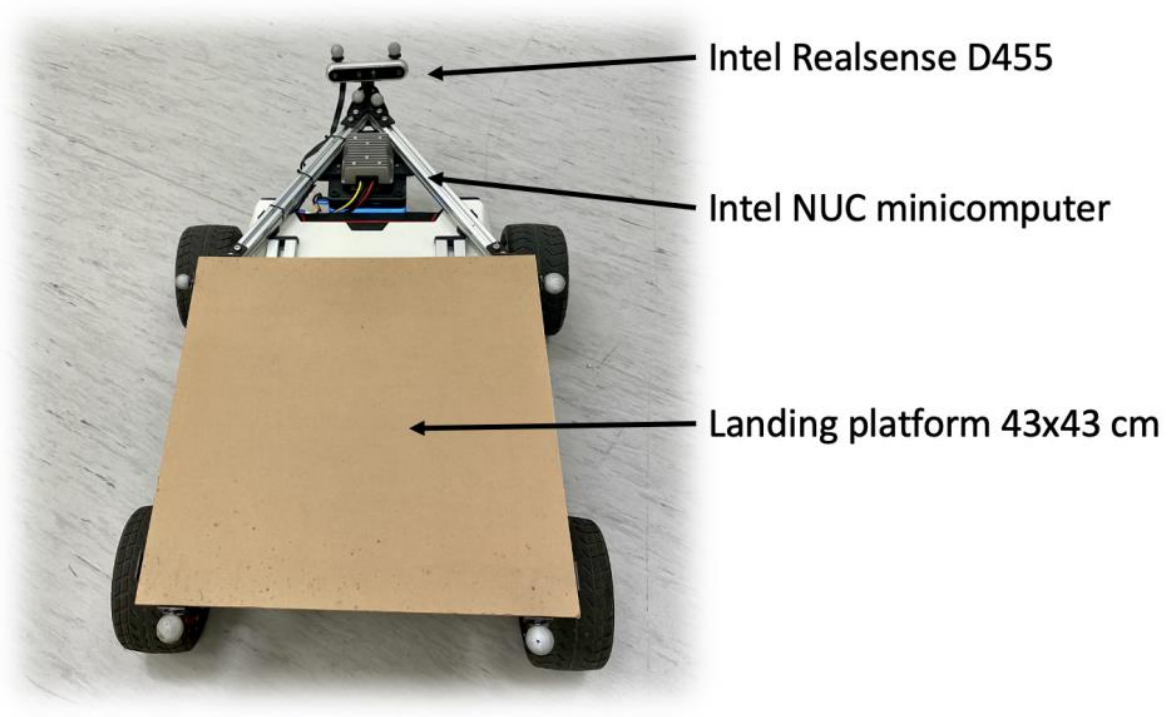

Figure 4. The hardware setup of the landing platform UGV. 
Figure 5 shows the UAV applied in the flight tests, which is a typical X-type quadrotor with a diameter of $210 \mathrm{~mm}$. The four motors are EMAX RS2205 (EMAX model, China) controlled through four T-MOTOR F35A (T-MOTOR, China) Electronic Speed Controllers (ESC). The ESCs are commanded by the flight controller, which is a mRo Pixracer (Mayan Robotics LLC, USA). The flight controller is equipped with several onboard sensors, including a gyroscope, accelerometer, compass, and barometer to self-control its attitude during the flight. In addition, Pixracer is also equipped with an onboard WIFI module, which can directly connect to the ROS system with MAVROS. This small quadrotor is also equipped with a front facing 3D-printed ArUco plate $(45 \times 45 \mathrm{~mm})$ to be recognized by the vision-based system.

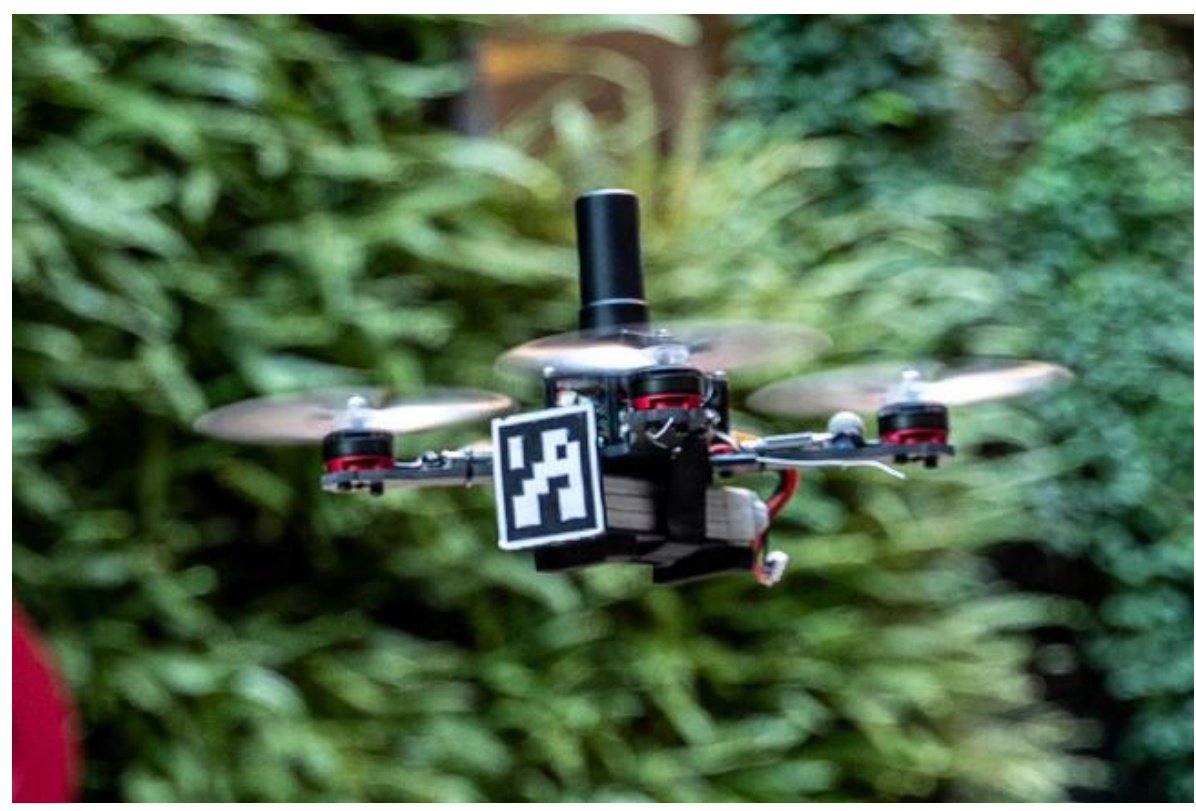

Figure 5. The hardware setup of the quadrotor UAV.

\subsection{Test Environment}

In Figure 6 the framework of the experimental system is demonstrated. The landing platform and UAV are connected through a WIFI access point to the ground station computer, which also acts as the major computational center. This ground station computer is equipped with an Intel I7-10700KF (Intel Corporation, USA) processor and two NVIDIA RTX 3090 (Nvidia Corporation, USA) Graphics Processing Units (GPU), ensuring the computational capacity of the system.

The flight experiments are first held in an indoor laboratory, which is equipped with a VICON (Vicon Motion Systems, UK) motion capture system providing the real-time ground truth information to the ground station computer. Then the experiments are moved to the outdoor for testing the system in the real-world environment. During the outdoor test, individual GPS modules are placed on both UGV and UAV, such that they can be guided roughly by the GPS coordinates information.

Notably, the current setup only serves as a demonstration. The UGV, which is the current experimental platform, is unable to carry a full-size computer onboard. The ground station computer is remotely connecting to UAV and UGV through a high bandwidth wireless network. In future real applications, the ground station can be directly installed at the landing platform with multiple cameras linking to it, providing seamless and powerful computational power. 


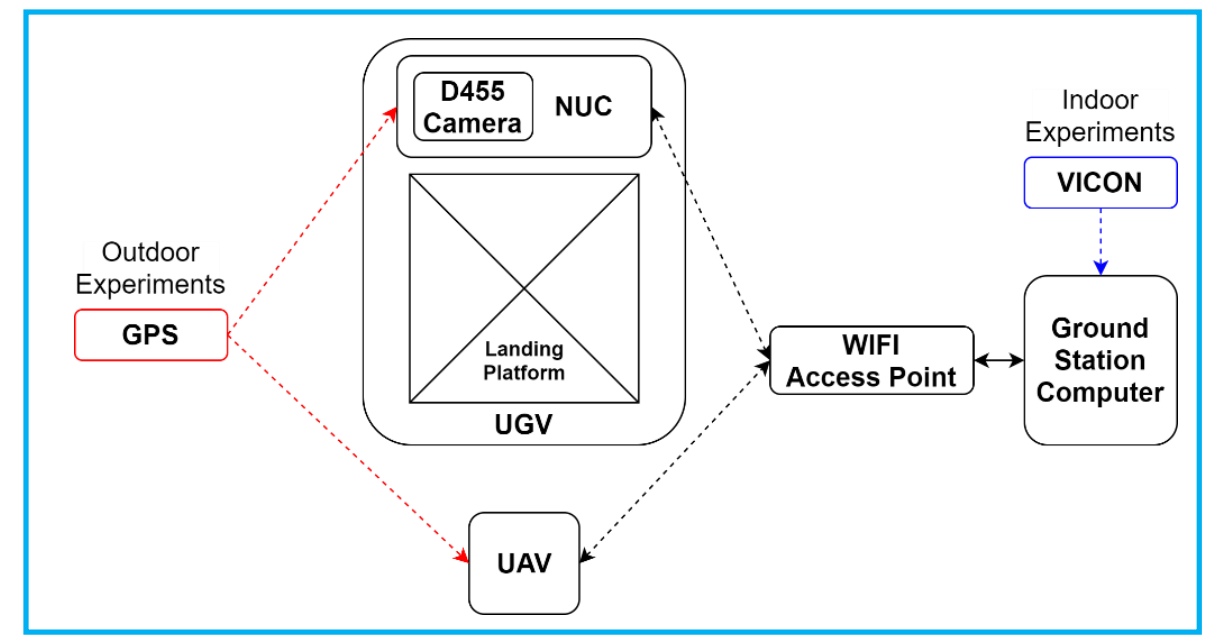

Figure 6. The framework of the experimental setup.

4. Results and Discussion

In this section, a series of indoor experiments are presented and discussed, followed by that of the outdoor flight experiments.

\subsection{Indoor Experiments Results}

This section summarizes the experiment results that are conducted in a controlled indoor environment. Ground truth data from the VICON system is used to validate the proposed vision-based position estimation result. The proposed trajectory generating method and conventional vertical landing method are also compared.

\subsubsection{Vision-Based Position Estimation Validation}

In this section, the task in "Second stage precise - following" in section 2.3.2 from the finite state machine is conducted with the quadrotor UAV in the controlled laboratory. The UGV is commanded to move forward at a constant velocity.

The first flight test shown in Figure 7 demonstrates the result of the quadrotor UAV landing on the moving UGV. The UAV is equipped with an ArUco marker, and it performs the precise following task. Some unwanted straight horizontal lines are shown in the ArUco state (green line), especially from $18.5 \mathrm{~s}$ to $22.5 \mathrm{~s}$. Also, its performance in the $z$-direction is the worst among all directions throughout the whole experiment. These straight horizontal lines indicate that, at these time segments, the ArUco marker on the UAV is not recognized by the vision position detector on the UGV. On the other hand, the Yolo (blue line) can keep a very close distance to the ground truth (black dash line), which shows that the proposed learning-based system has a much more stable and accurate recognition ability. With the help of the Yolo, the overall estimation (red line) is able to maintain a tight track to the ground truth throughout the experiment period. This result shows that it is critical to combine the learning-based recognition into the system. 

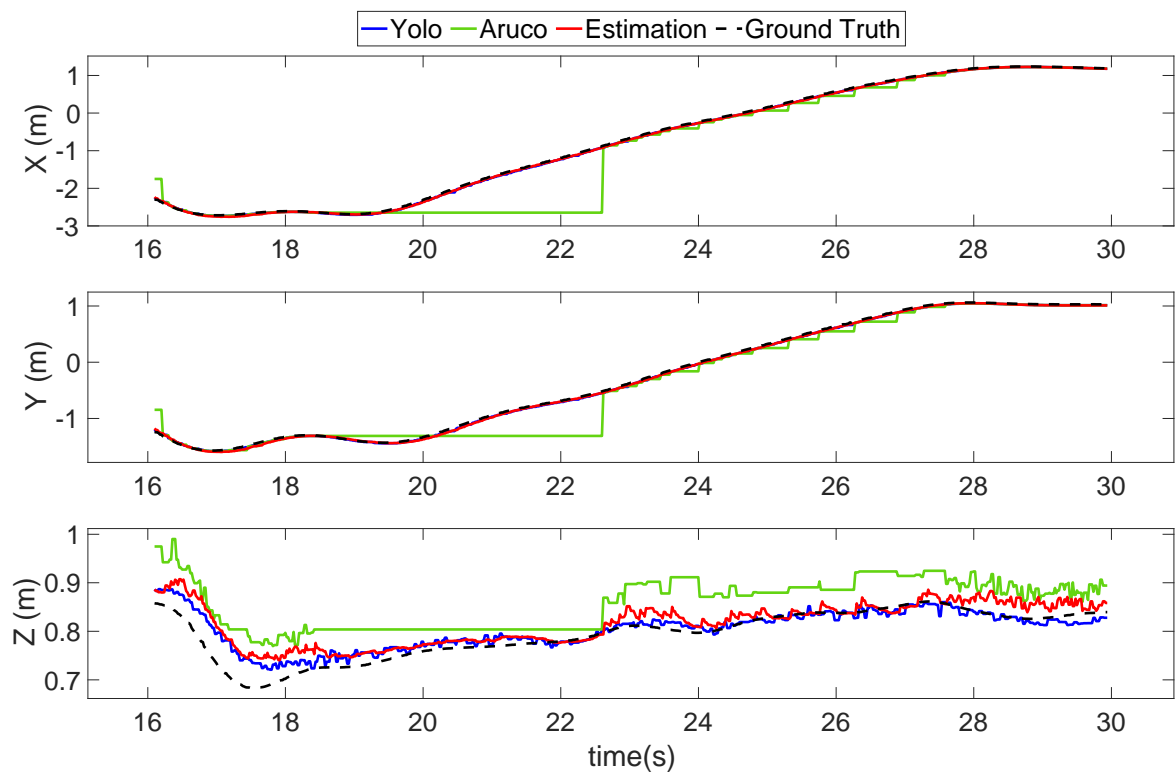

Figure 7. Position estimation result by using the proposed vision-based system and sensor fusion method in a moving platform following mission.

Table 1 displays the root mean square error (RMSE) and mean absolute error (MAE) of the estimated position to the ground truth, which shows the overall performance of the position estimator is stable and precise enough to perform the auto landing task. The overall error is less than $4 \mathrm{~cm}$ compared to the size of the landing platform of $43 \mathrm{~cm}$. Therefore, the proposed estimation system is precise enough to perform the autonomous landing task.

Table 1. Calculated RMSE and MAE of UAV position estimation

\begin{tabular}{cccc}
\hline Error Evaluation & $\mathrm{X}(\mathrm{m})$ & $\mathrm{Y}(\mathrm{m})$ & $\mathrm{Z}(\mathrm{m})$ \\
\hline RMSE & 0.0335 & 0.0275 & 0.0312 \\
MAE & 0.0298 & 0.0247 & $0.0237^{\vee}$ \\
\hline
\end{tabular}

\subsubsection{Landing Trajectories Experiments}

In this section, the UAV is commanded to perform two kinds of landing trajectories toward a non-moving landing platform. These experiments are also conducted in the controlled VICON laboratory, where the position reference is directly detected and provided by the VICON system for accurate validation.

Figure 8 shows the first experiment, which is a conventional vertical landing trajectory. As the figure shows, while the UAV starts to move downward vertically, the horizontal position started to wobble, and it is unable to precisely maintain the commanded setpoint. Furthermore, at the last 15 centimeters $(29 \mathrm{~s} \sim 34 \mathrm{~s})$ before touching down on the $\mathrm{z}$-direction, the UAV faces large difficulty to keep descending since the ground effect begins to take effective. At this certain time of period, the horizontal position of $\mathrm{x}$ - and $\mathrm{y}$ direction are even more out of control. 

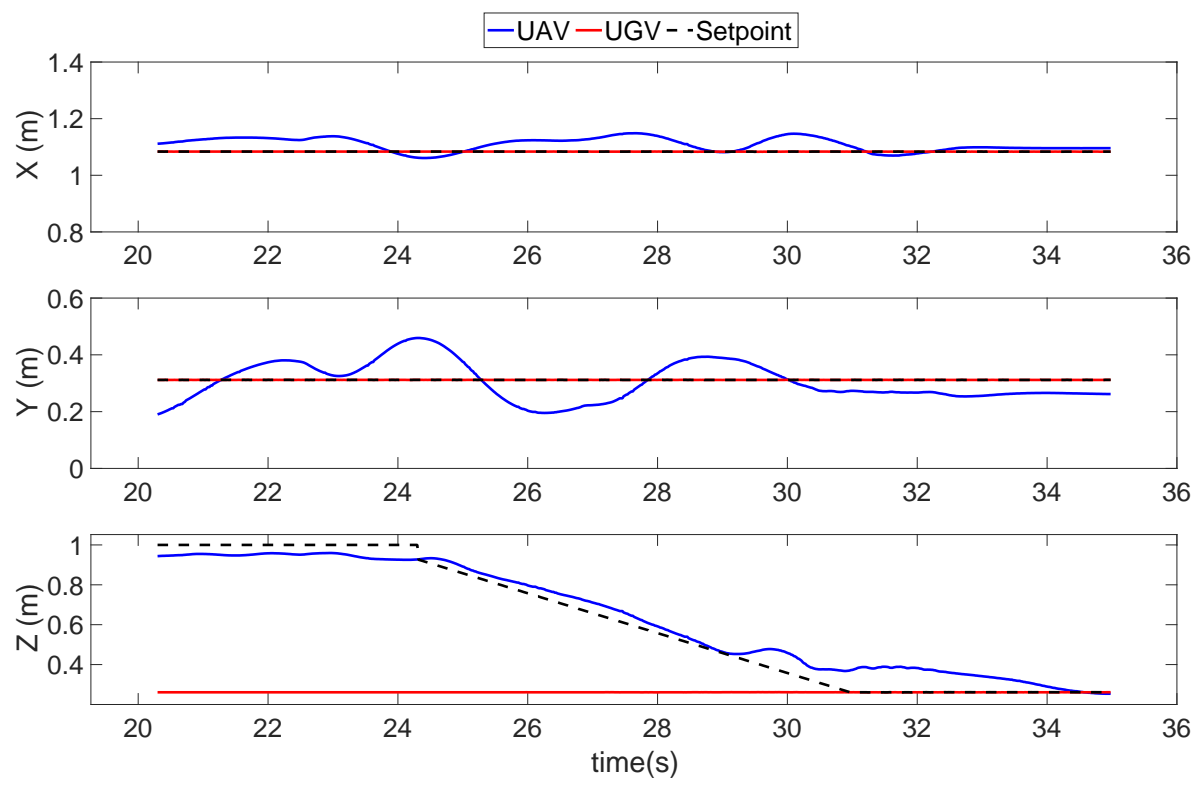

Figure 8. The position result of a quadrotor UAV conducting a conventional vertical landing trajectory.

On the contrary, the experiment in Figure 9 applies the proposed ground-effect free trajectory. The UAV enters the landing state at an initial position of 1 meter away to the AGV in the $x$-direction and 0.25 meter in the y-direction. Then the UAV starts to follow the gliding and landing trajectory, which is descending in the z-direction while also approaching the AGV in the $\mathrm{x}$ - and $\mathrm{y}$-direction. We can see that the effectiveness of the ground effect is gradually reduced at the final period. Additionally, the horizontal position performance is also outperformed from the previous result in Figure 8.
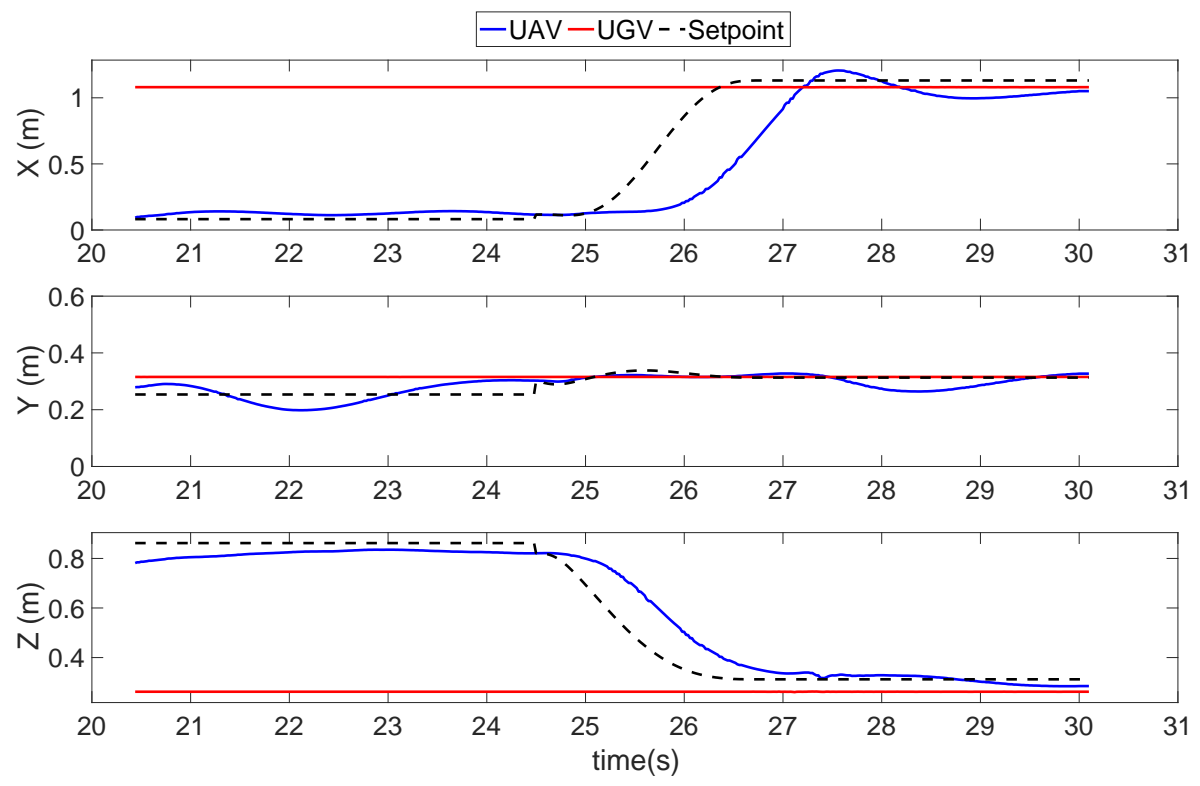

Figure 9. The position result of a quadrotor UAV conducting the proposed ground effect free landing trajectory.

\subsection{Outdoor Experiments Results}

This experiment tests the system in the real-world environment. In this flight test, the quadrotor UAV is trying to land on the constant moving speed UGV, and the functionality 
of the state machine is validated. The position of the UGV is collected by the onboard GPS and the position of UAV are from the proposed position estimating system.

In Figure 10, we can see that the UAV does three tries to approach the landing pad, which are 20.8 23.3s, 26.3 28.4s, and 31.5 35s. In the first try, the state machine enters the Third stage Ground Effect Free Trajectory following at 20.8 s. During the approaching maneuver (20.8 23.3 s), the position of $x$ - and z-direction are well followed with only one second in delay. However, there is an overshoot in the y-direction at 24s (red arrow), which triggers the failsafe and rewinds the state machine to the Second stage - precise following.

The second attempt (26.3 28.4 s) shows a similar phenomenon; however, an overshoot happens in the z-direction at 28.5s (green arrow), which means the altitude of the UAV drops beneath the expectation level The state machine also switches back to the Second stage right away. In the third attempt (31.5 35s), the UAV finally meet all the safety requirements and land safely on the landing platform.
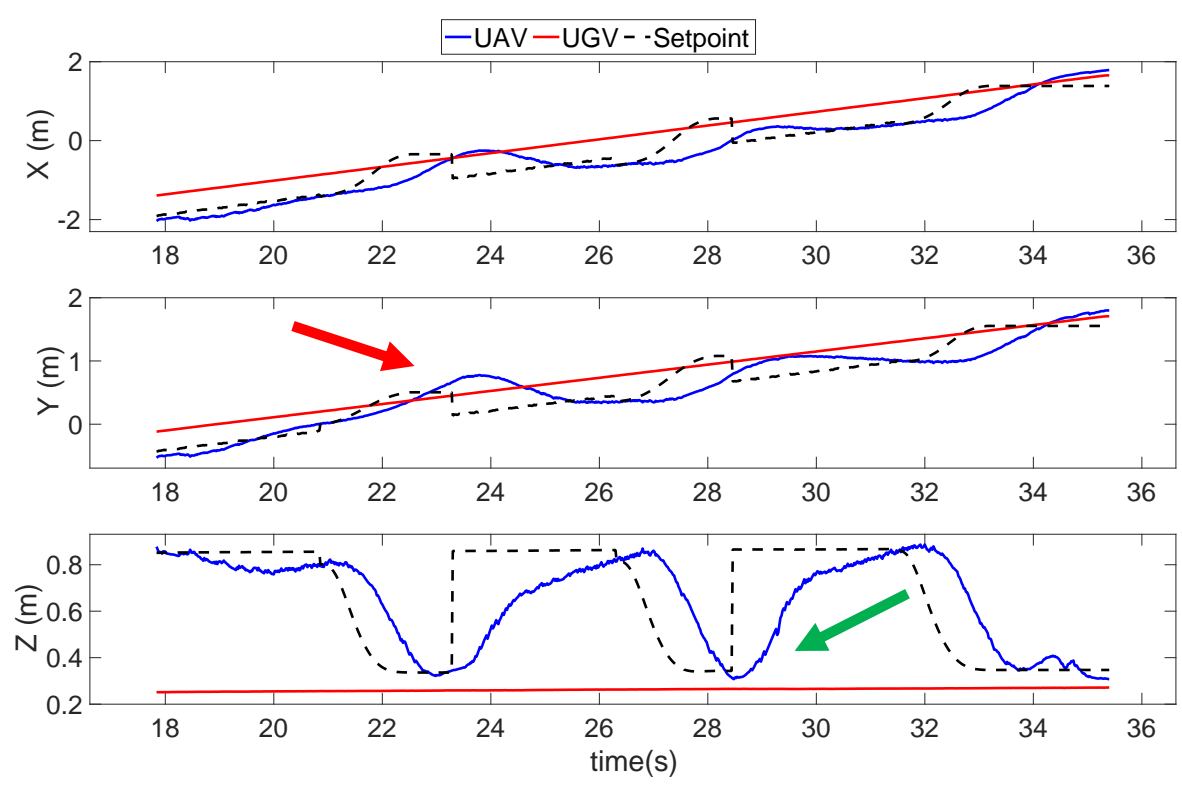

Figure 10. The position result of the UAV landing on a moving platform in an outdoor experiment.

Furthermore, Figure 11 shows the result from the vision system. We can see that especially during the second approach (27 30 s), in which the UAV is very close to the camera, the YOLO (blue line) starts to lose track of the UAV. In the meantime, the ArUco is functioning appropriately, which secures the position estimation result on track. Hence, this result shows the importance of sensor fusion and redundancy in the vision system. 

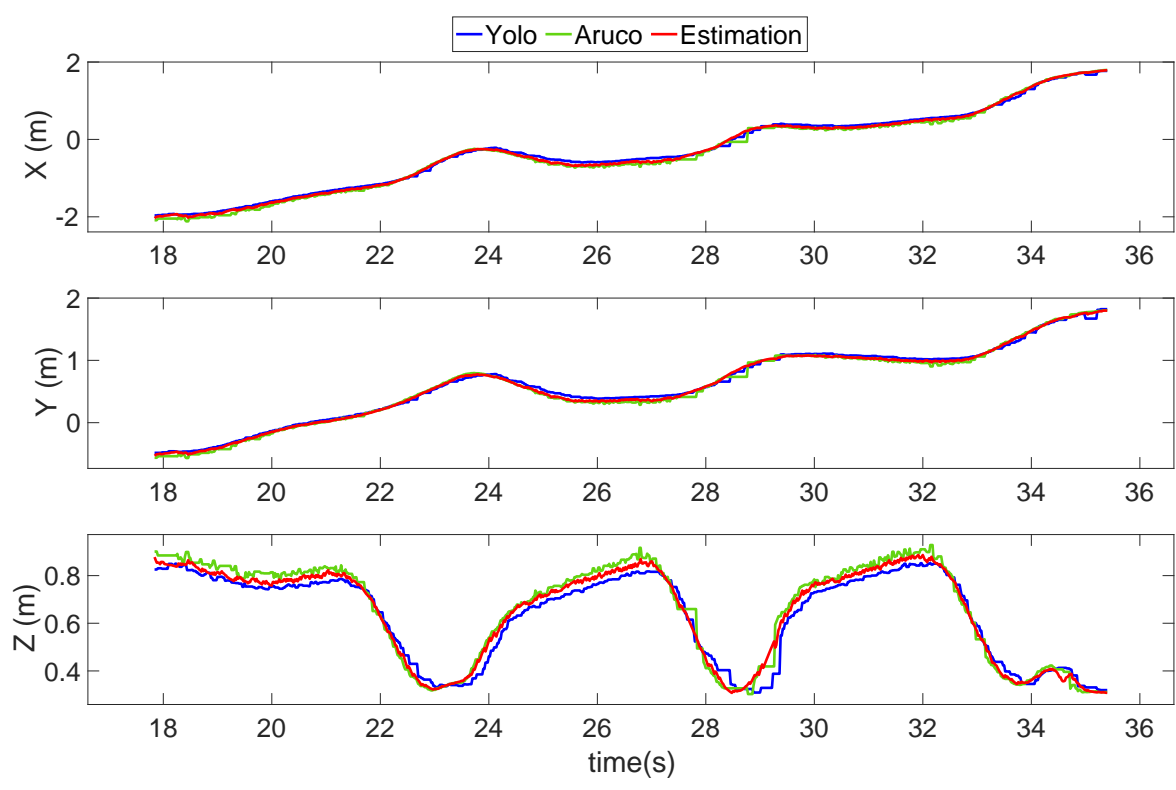

Figure 11. Position estimation result of the UAV landing on a moving platform in an outdoor experiment.

\section{Conclusions}

In this study, the objective is to develop a proactive guidance system for UAVs to land accurately on a moving platform. With the proposed system structure, the of UAV's onboard instruments can be reduced. Two sets of vision-based position detection modules are firstly introduced, followed by a sensor fusion and estimation technics aiming at the performance enhancement of position estimation. After that, a finite state machine system with an optimal trajectory generating method is designed to accomplish this landing guidance task.

Flight experiments are taken in both indoor and outdoor environments. The visionbased estimation result is first validated and tested in a controlled laboratory. The result proves the reliability of the vision-based system. In the landing trajectory experiments, a feasible way to prevent the unstable movement caused by ground effect during the landing progress is presented. At last, the system is tested in a real-world environment with the UAV safely landed.

To obtain a further improvement of the UAV landing guidance system, attention will be focused on the estimation of the relative movement of the UAV and the landing platform. In marine applications, the landing platform is believed to be heaving and shaking on the waves. The system will need to be able to measure and estimate the relative movement to achieve a safe landing in a more chaotic environment.

Author Contributions: Conceptualization, C.-W.C.; methodology, C.-W.C. and L.-Y.L.; software, C.-W.C. and L.-Y.L. and Y.F.; validation, C.-W.C. and L.-Y.L. and H.C.C; formal analysis, C.-W.C. and Y.F.; data curation, C.-W.C. and H.C.C and Y.F.; writing - original draft preparation, C.-W.C.; writing, review and editing, W.Z. and A.-S.Y. and C.-Y.W.; supervision, W.Z. and A.-S.Y. and C.Y.W.; All authors have read and agreed to the published version of the manuscript.

Funding: This work was supported by the Innovation and Technology Commission of Hong Kong under grant number ITT/027/19GP.

Data Availability Statement: Not applicable.

Conflicts of Interest: The authors declare no conflict of interest. 


\section{References}

[1] H. Zhang, L. Wang, T. Tian, and J. Yin, "A Review of Unmanned Aerial Vehicle Low-Altitude Remote Sensing (UAV-LARS) Use in Agricultural Monitoring in China," Remote Sensing, vol. 13, no. 6, p. 1221, 2021. [Online]. Available: https://www.mdpi.com/2072-4292/13/6/1221.

[2] J. Liu, J. Xiang, Y. Jin, R. Liu, J. Yan, and L. Wang, "Boost Precision Agriculture with Unmanned Aerial Vehicle Remote Sensing and Edge Intelligence: A Survey," Remote Sensing, vol. 13, no. 21, p. 4387, 2021. [Online]. Available: https://www.mdpi.com/2072-4292/13/21/4387.

[3] W.-C. Chiang, Y. Li, J. Shang, and T. L. Urban, "Impact of drone delivery on sustainability and cost: Realizing the UAV potential through vehicle routing optimization," Applied energy, vol. 242, pp. 1164-1175, 2019.

[4] A. M. Ham, "Integrated scheduling of m-truck, m-drone, and m-depot constrained by time-window, drop-pickup, and mvisit using constraint programming," Transportation Research Part C: Emerging Technologies, vol. 91, pp. 1-14, 2018.

[5] R. Stone and G. Clarke, "Optimization of transition manoeuvres for a tail-sitter unmanned air vehicle," in UAV)," Australian Aerospace International Congress [online paper], http://www. aeromech. usyd. edu. au/uav/twing/pdfs/AIAC_paper_final. pdf, Paper 105, 2001: Citeseer.

[6] DHL, "Unmanned Aerial Vehicles in Logistics." [Online]. Available: https://www.dhl.com/global-en/home/insights-andinnovation/thought-leadership/trend-reports/unmanned-aerial-vehicles.html

[7] "Amazon Prime Air." https://www.amazon.com/Amazon-Prime-Air/b?ie=UTF8\&node=8037720011 (accessed.

[8] J. Leighton, "System design of an unmanned aerial vehicle (UAV) for marine environmental sensing," MASSACHUSETTS INST OF TECH CAMBRIDGE DEPT OF MECHANICAL ENGINEERING, 2013.

[9] İ. Bayırhan and C. Gazioğlu, "Use of Unmanned Aerial Vehicles (UAV) and Marine Environment Simulator in Oil Pollution Investigations," in International Symposium on Applied Geoinformatics (ISAG-2019), 2019, pp. 1-6.

[10] G. Xu, W. Shen, and X. Wang, "Applications of wireless sensor networks in marine environment monitoring: A survey," Sensors, vol. 14, no. 9, pp. 16932-16954, 2014.

[11] G. Mills and G. Fones, "A review of in situ methods and sensors for monitoring the marine environment," Sensor Review, 2012.

[12] A. Klimkowska, I. Lee, and K. Choi, "Possibilities of UAS for maritime monitoring," The International Archives of Photogrammetry, Remote Sensing and Spatial Information Sciences, vol. 41, p. 885, 2016.

[13] A. J. Healey, D. Horner, S. Kragelund, B. Wring, and A. Monarrez, "Collaborative unmanned systems for maritime and port security operations," NAVAL POSTGRADUATE SCHOOL MONTEREY CA CENTER FOR AUTONOMOUS UNDERWATER ..., 2007.

[14] U. Dahana and R. Gurning, "Maritime aerial surveillance: integration manual identification system to automatic identification system," in IOP Conference Series: Earth and Environmental Science, 2020, vol. 557, no. 1: IOP Publishing, p. 012014.

[15] N. Trigoni and S. Waharte, "Supporting search and rescue operations with uavs," 2010.

[16] E. Ebrahimi-Oskoei, "Swarm of UAVs: Search \& Rescue Operationin Chaotic Ship Wakes," 2014.

[17] D. W. Schuldt and J. Kurucar, "Maritime Search and Rescue via Multiple Coordinated UAS," MIT Lincoln Laboratory Lexington United States, 2016.

[18] S. Losey, "DARPA nabs Gremlin drone in midair for first time," in Defense News, ed. "https://www.defensenews.com/unmanned/2021/11/05/darpa-nabs-gremlin-drone-in-midair-for-first-time/"

[19] N. Xuan-Mung, S. K. Hong, N. P. Nguyen, and T.-L. Le, "Autonomous quadcopter precision landing onto a heaving platform: New method and experiment," IEEE Access, vol. 8, pp. 167192-167202, 2020.

[20] A. Borowczyk, D.-T. Nguyen, A. Phu-Van Nguyen, D. Q. Nguyen, D. Saussié, and J. Le Ny, "Autonomous landing of a multirotor micro air vehicle on a high velocity ground vehicle," Ifac-Papersonline, vol. 50, no. 1, pp. 10488-10494, 2017. 
[21] A. Keipour et al., "Visual Servoing Approach for Autonomous UAV Landing on a Moving Vehicle," arXiv preprint arXiv:2104.01272, 2021.

[22] M. Demirhan and C. Premachandra, "Development of an automated camera-based drone landing system," IEEE Access, vol. 8, pp. 202111-202121, 2020.

[23] D. Lee, T. Ryan, and H. J. Kim, "Autonomous landing of a VTOL UAV on a moving platform using image-based visual servoing," in 2012 IEEE international conference on robotics and automation, 2012: IEEE, pp. 971-976.

[24] W. Liu et al., "Ssd: Single shot multibox detector," in European conference on computer vision, 2016: Springer, pp. 21-37.

[25] J. Redmon, S. Divvala, R. Girshick, and A. Farhadi, "You only look once: Unified, real-time object detection," in Proceedings of the IEEE conference on computer vision and pattern recognition, 2016, pp. 779-788.

[26] Y. Feng, K. Tse, S. Chen, C.-Y. Wen, and B. Li, "Learning-Based Autonomous UAV System for Electrical and Mechanical (E\&M) Device Inspection," Sensors, vol. 21, no. 4, p. 1385, 2021.

[27] N. Metni and T. Hamel, "A UAV for bridge inspection: Visual servoing control law with orientation limits," Automation in Construction, vol. 17, no. 1, pp. 3-10, 2007/11/01/ 2007, doi: https://doi.org/10.1016/j.autcon.2006.12.010.

[28] L.-Y. Lo, C. H. Yiu, Y. Tang, B. Li, and C.-Y. Wen, "Dynamic Object Tracking on Autonomous UAV System: For Surveillance Applications," 2021.

[29] L. Wang and X. Bai, "Quadrotor autonomous approaching and landing on a vessel deck," Journal of Intelligent $\mathcal{E}$ Robotic Systems, vol. 92, no. 1, pp. 125-143, 2018.

[30] A. Paris, B. T. Lopez, and J. P. How, "Dynamic landing of an autonomous quadrotor on a moving platform in turbulent wind conditions," arXiv preprint arXiv:1909.11071, 2019.

[31] Z. Wang, X. Zhou, C. Xu, J. Chu, and F. Gao, "Alternating minimization based trajectory generation for quadrotor aggressive flight," IEEE Robotics and Automation Letters, vol. 5, no. 3, pp. 4836-4843, 2020. 